\title{
El significado de las rocas: El debate de Kuhn y Taylor sobre la base hermenéutica de la ciencia
}

\section{The meaning of the rocks: Kuhn and Taylor's debate about the hermeneutic foundation of science}

\author{
LEONARDO DÍAZ \\ Universidad Autónoma de Santo Domingo (República Dominicana)
}

Recibido: 8-6-2011

Aprobado definitivamente: 10-10-2012

\begin{abstract}
RESUMEN
A finales de los años 80s, Thomas Kuhn y Charles Taylor fueron invitados a un debate en La Salle University. Taylor defendió que las ciencias naturales no son ciencias hermenéuticas, pues se fundamentan en datos puros, carentes de significado. Kuhn rechazó la tesis de la existencia de datos puros, sosteniendo que las ciencias naturales operan con significados y poseen una base hermenéutica. En la postura de Kuhn pueden apreciarse ambivalencias como resultado de sus viejos compromisos teóricos con el proyecto explicativo formulado en La estructura de las revoluciones científicas y como mostraré, vinculado a la existencia de una tensión entre dos perspectivas filosóficas sobre la ciencia.
\end{abstract}

PALABRAS CLAVE

HERMENÉUTICA, CIENCIA NORMAL, CIENCIA REVOLUCIONARIA, TENSIÓN, CIENCIAS HUMANAS

(C) Contrastes. Revista Internacional de Filosofía, vol. XVIII (2013), pp. 285-302. ISSN: 1136-4076 Licenciatura de Filosofía, Universidad de Málaga, Facultad de Filosofía y Letras Campus de Teatinos, E-29071 Málaga (España) 


\begin{abstract}
By the end of the 1980s, Thomas Kuhn and Charles Taylor participated in a debate at La Salle University. Taylor defended that natural sciences are not hermeneutical sciences, since they are based on the pure, meaningless data. Kuhn rejected the thesis of the existence of pure data, arguing that natural sciences work with meanings and have a hermeneutic foundation. Kuhn's position presents ambivalences as a result of his former theoretical commitments with the explicative project formulated in The Structure of the Scientific Revolutions and as I will show, linked to the existence of a tension between two philosophical perspectives on science.
\end{abstract}

KEYWORDS

HERMENEUTICS, NORMAL SCIENCE, REVOLUTIONARY SCIENCE, TENSION, HUMAN SCIENCES

\title{
I. INTRODUCCIÓN
}

En el verano de 1988, Thomas Kuhn y Charles Taylor participaron en el Curso de verano sobre la Interpretación en el Summer Institute of Interpretation (NEH). Taylor era un defensor de la vieja demarcación diltheyana entre las ciencias naturales y las ciencias del espíritu, mientras Kuhn se oponía a los fundamentos de esta demarcación.

Para Taylor las ciencias humanas estudian los significados, las acciones humanas y su intencionalidad, mientras las ciencias naturales estudian datos puros independientes del contexto histórico y cultural. Por consiguiente, no pueden considerarse ciencias hermenéuticas. ${ }^{1}$

Esta postura contrastaba con la imagen de las ciencias naturales que Kuhn había presentado en La estructura de las revoluciones científicas. La idea de que no existen datos desvinculados de las creencias y que éstos datos se conforman a partir de los sistemas de prácticas de las comunidades científicas desarrolladas en el tiempo y la cultura, así como el planteamiento de la existencia de la incon-

1 La tesis de que las ciencias humanas, a diferencia de las ciencias naturales, no se fundamentan en datos puros se relaciona con el dogma empirista de la distinción entre el lenguaje teórico y el lenguaje observacional. Según este supuesto, en las ciencias naturales existen datos puros, porque existen observaciones no conceptualizadas. El carácter no conceptualizado de los datos de las ciencias naturales les permite a quienes practican estas ciencias no contaminar con sus particulares creencias o ideologías la descripción de la realidad. Siguiendo con esta perspectiva, en las ciencias humanas no es posible hablar de datos puros, porque los mismos están conceptualizados. Para una panorámica bastante extensa sobre la evolución del problema, cf. L. Olivé y A.R.Pérez Ransanz (eds.) 1998. 
mensurabilidad $^{2}$ entre distintos sistemas de prácticas, son los pilares de su obra clásica de 1962 y de su pensamiento posterior. Si lo que distingue a las ciencias naturales de las humanas es el hecho de que las primeras poseen datos puros y carecen de interpretación histórica y cultural, entonces, una consecuencia de La estructura de las revoluciones científicas es que no hay lugar para trazar la línea demarcatoria y las ciencias naturales pueden ser consideradas igualmente como «ciencias hermenéuticas».

Pero a pesar de su postura con respecto a la naturaleza de los objetos de las ciencias naturales, Kuhn fue ambiguo con respecto al problema de la distinción con las ciencias humanas. Estaba claro que la distinción diltheyana no lo satisfacía, pero no sabía dónde trazar la demarcación y por momentos parecía vacilar sobre el hecho de si era necesario hacerlo. Con este estado de la cuestión, Kuhn y Taylor son invitados por La Salle University para debatir sus posturas.

\section{KUHN VERSUS TAYLOR:}

DEL SIGNIFICADO DE LOS TEXTOS AL SIGNIFICADO DE LAS ROCAS

El 11 de febrero de 1989, casi tres décadas después de protagonizar junto a Karl Popper uno de los debates de mayor impacto en la filosofía de la ciencia del siglo XX, Thomas Kuhn asistió a La Salle University para, junto Charles Taylor, participar en una mesa redonda sobre la distinción entre las ciencias naturales y las humanas. ${ }^{3}$

Taylor (1985) había escrito cuatro años antes, un artículo titulado «Interpretation and the sciences of man». Según Taylor, una ciencia hermenéutica -como lo son las ciencias humanas-posee un objeto que debe ser descrito en términos de sentido y sin sentido, de coherencia e incoherencia, debiendo incorporar una distinción entre el significado y su expresión. Para que esto sea posible se necesita

2 Aunque Kuhn emplea el concepto de inconmensurabilidad una docena de veces en $L a$ estructura de las revoluciones científicas, no lo define claramente. Esta es una de las razones de que su uso generara una serie de malentendidos que Kuhn intentó solventar en su obra posterior. H. Sankey distingue tres períodos en el uso kuhniano del término. El primero implica el uso de la noción entendida como incompatibilidad entre escuelas desde el punto de vista conceptual, observacional y metodológico. El segundo período se relaciona con una noción semántica de la inconmensurabilidad, mientras el tercer período alude al término implicando un problema de fallos de traducción focalizados en un subconjunto de términos que constituyen dos teorías en pugna. Sobre el uso kuhniano de la inconmensurabilidad, cf. T. Kuhn 1970, pp.4, 103, 112 , 148-150, 157, 165, 175, 198, 200. Sobre la evolución del concepto de inconmensurabilidad en la obra de Kuhn, cf. H. Sankey 1993, pp. 775-791. Para críticas al concepto kuhniano de inconmensurabilidad, cf. A. Bird 2000, cap. 5; S. Gattei 2008, cap. 3.

3 Según atestigua el mismo Kuhn, Taylor tuvo que abandonar repentinamente la mesa de discusión, por lo que su respuesta a las posiciones de Taylor se expuso en ausencia de éste. Cf. T. Kuhn 2000a, pp. 216-223. 
un sujeto. Por su parte, las ciencias naturales no son ciencias hermenéuticas, debido a que carecen de estas características. Según Taylor:

Podemos hablar del sentido o de la coherencia, así como de sus concretizaciones, en conexión con fenómenos tales como la Gestalt, configuraciones en la formación de rocas, o cristales de nieve, donde la noción de expresión no tiene una justificación real. De lo que se carece aquí es de la noción de un sujeto para quien existan estos significados. (Ch. Taylor 1985, p.16). ${ }^{4}$

Por tanto, los objetos de las ciencias naturales son asignificativos, entidades del mundo. «Las rocas carecen de significado».

La postura de Kuhn se encontraba aparentemente en las antípodas de esta imagen demarcatoria entre las ciencias. Criticó a Taylor por esta perspectiva así como por la postura de éste sobre el carácter ahistórico y no cultural de los objetos de las ciencias naturales. Por el contrario, para Kuhn (2000a) las ciencias naturales están determinadas temporal y culturalmente, llegando a sostener que las ciencias naturales tienen una base hermenéutica.

También, Taylor sostuvo que para considerar una ciencia como hermenéutica, ésta debe poseer datos con significado, tal y como ocurre con los comportamientos de los seres humanos. Sus significados se constituyen para un sujeto en un campo determinado. Estos significados son autodefiniciones parciales y por tanto, son ya interpretaciones. ${ }^{5}$ Finalmente, estos significados pueden ser re-expresados o reformulados explícitamente por una ciencia hermenéutica.

Al igual que otros filósofos de la tradición hermenéutica, como Heidegger (1974) y Gadamer (1977), Taylor (1985) afirma que el proceso de comprensión característico de las ciencias humanas se desarrolla dentro de un círculo hermenéutico. Tratamos de comprender textos o expresiones apelando como fundamento de esta comprensión a la lectura de otros textos. Se da una relación en que la comprensión general depende de nuestra apelación a las experiencias parciales que lo constituyen. Estas a su vez adquieren sentido en sus interrelaciones y en su relación con la totalidad del texto.

Del mismo modo, la totalidad de los actos humanos son comprendidos dentro de un círculo hermenéutico. Los términos que aluden a determinadas emociones o estados de ánimo remiten a situaciones específicas. A la vez, estas situaciones son comprensibles a partir de las experiencias emocionales vividas.

4 Esta y todas las traducciones del artículo son del autor.

5 Taylor considera que debemos pensar el ser humano como un «animal autointerpretativo». En términos de las ciencias humanas no existe algo como una estructura de significados independiente del proceso mismo mediante el cual el ser humano se autodefine y se autocomprende. Cf. Ch. Taylor: Op. cit. pp. 55-56. 
Los términos que se refieren a estados emocionales son explicados a partir de otros términos que son comprendidos en relación con su referencia a los estados emocionales que intentan comprender. Y la comprensión de esos conceptos se articula en torno a una estructura lingüística que implica un conjunto de acciones y significados compartidos.

Los estados emocionales, actos y significados son inseparables de las categorizaciones marcadas por el lenguaje compartido por los integrantes de una determinada cultura.

$\mathrm{Al}$ asumir la tesis de la existencia de datos puros en las ciencias naturales, Taylor negaba la existencia de un círculo hermenéutico en las mismas. No existe ninguna circularidad interpretativa allí donde partimos de algo dado, desprovisto de preconcepciones o prejuicios. Si en las ciencias naturales no existe ninguna estructura pre-interpretativa que anteceda a la configuración de los datos, entonces no puede hablarse de interpretación en términos hermenéuticos. ${ }^{6}$

Por consiguiente, no existe un proceso permanente de interpretación o de lecturas que remita a otros términos y lecturas. El proceso concluye con la remisión a una instancia no conceptualizada. Esta tesis se relaciona con el planteamiento de la ahistoricidad de los datos. La entidades del mundo natural carecen de historia y cultura, por ser ajenas a las interpretaciones humanas que sí están condicionadas por ellas.

Kuhn no se refiere explícitamente al problema del círculo hermenéutico pero puede inferirse su postura al respecto partiendo de sus planteamientos en La estructura de las revoluciones científicas sobre el carácter conceptualmente predeterminado de los datos, y por tanto, a la inexistencia de datos puros:

Lo que un hombre ve depende tanto de lo que observa como también de lo que le ha enseñado a ver su experiencia visual y conceptual previa. En ausencia de tal entrenamiento, sólo puede existir «una lujuriante y zumbante confusión», para decirlo con las palabras de William James. (T. Kuhn 1970, p. 113).

Aunque posteriormente a la publicación de La estructura la noción de paradigma no siguió desempeñando la misma relevancia que en los años 60s, fue coherente con la concepción de que las observaciones requieren presuposiciones, no existen datos puros. Décadas después, en la mesa redonda del debate con Taylor, afirmó:

No comparé ni puedo comparar nuestros cielos con los de los japoneses, pero dije e insistiré aquí que los nuestros son diferentes de los cielos de los antiguos griegos

6 En la tradición hermenéutica la interpretación siempre implica una estructura precomprensiva, un sistema de anticipaciones y prejuicios que condicionan la comprensión. Cf. al respecto, H. Gadamer 1977, pp.336-337. 
[...]. Nuestras taxonomías celestiales son sistemáticamente distintas. Para los griegos, los objetos celestes estaban divididos en tres categorías: estrellas, planetas y meteoros. Nosotros tenemos categorías con estos nombres, pero lo que los griegos incluían con las suyas era muy diferente de lo que nosotros incluimos con las nuestras. El Sol y la Luna iban dentro la misma categoría que Júpiter, Marte, Mercurio, Saturno y Venus. Para ellos, estos cuerpos eran semejantes entre sí y diferentes de los miembros de las categorías «estrella» y «meteoro». Por otra parte, situaban la Vía Láctea, que para nosotros está poblada de estrellas, en la misma categoría que al arco iris, los anillos que hay alrededor de la Luna, las estrellas fugaces y otros meteoros. Hay otras diferencias de clasificación similares. Así pues, cosas semejantes en un sistema eran diferentes en el otro. Desde la antigüedad griega, la taxonomía de los cielos, los patrones de similaridad y diferencia celestiales han cambiado sistemáticamente. (T. Kuhn 2000a, pp. 218-219).

En La estructura de las revoluciones científicas, Kuhn defendió la teoría de la percepción de Norwood Hanson. La tesis de Hanson (1977), conocida como la tesis de la «carga teórica de las observaciones», implica un vínculo indisoluble entre las creencias y las observaciones de las comunidades científicas.

Tres décadas después, Kuhn siguió defendiendo el carácter cargado de los datos. Si los cielos de los griegos son distintos a los nuestros, es porque la observación de los cielos está indisolublemente vinculada y condicionada por las taxonomías de los observadores. Significa que los datos no son algo dado e inmutable, sino que los mismos están enmarcados dentro de un proceso de interpretación. El viejo planteamiento de Kuhn según el cual cuando cambian los marcos conceptuales de las comunidades científicas el mundo de los datos se modifica con ellos, ${ }^{7}$ permanece implícito en su debate con Taylor.

Al requerir de una estructura previa a los datos como condición de posibilidad de su constitución e interpretación, en las ciencias naturales puede hablarse de un círculo hermenéutico similar al de las ciencias humanas.

Kuhn (2000a) señala que desde una perspectiva empirista, un mero ejercicio de ostensión bastaría para lograr el acuerdo entre personas de culturas diferentes sobre cuáles son las características del objeto del mundo natural percibido.

Kuhn objeta la tesis del acuerdo observacional por ostensión, señalando que la misma no proporciona conocimiento «per se». Para ello, se requiere situar el

7 En La estructura de las revoluciones científicas, Kuhn se refiere a la noción de mundo en dos sentidos: como la realidad fenoménica y como la realidad nouménica. Mientras ésta es un mundo no conceptualizado y por tanto, incognoscible para las comunidades científicas, la realidad fenoménica es el mundo conceptualizado y por tanto, es accesible, pensable y cognoscible. Es el mundo de los datos que hace posible la ciencia. Para la noción de mundo en Kuhn, cf. P. Hoyninguen Huene 1998, pp. 31ss; L. Olivé 1998, pp 195-211. ; A.R. Pérez Ransanz y J.F.Álvarez 2004, pp. 495-517. 
objeto o el evento dentro de un marco conceptual general, dentro de una taxonomía previamente disponible que haga identificable, reconocible y comunicable el conocimiento del objeto. Esta taxonomía solo es disponible en el contexto de una cultura. Por tanto, las ciencias naturales son igualmente condicionadas desde el punto de vista histórico y cultural que las ciencias humanas:

No existe ningún conjunto de categorías neutral, independiente de la cultura, dentro del cual la población -sea de objetos o de acciones- pueda ser descrita; y en este sentido las ciencias naturales no tienen ninguna ventaja sobre las humanas. (T. Kuhn 2000a, p. 220).

Un año después del debate, Kuhn es más tajante al establecer las similitudes entre ciencia y literatura:

Los estudiantes de Literatura siempre han dado por sentado que la metáfora y sus mecanismos asociados (aquellos que modifican las interrelaciones entre las palabras) proporcionan la entrada a nuevos mundos y, de este modo, imposibilitan la traducción. Características similares se han atribuido, de manera amplia, al lenguaje de la vida política y, por algunos, al entero ámbito de las Ciencias humanas. Pero las ciencias naturales, al tratar objetivamente con el mundo real (como lo hacen), generalmente se han mantenido inmunes. Se piensa que sus verdades (y falsedades) trascienden los estragos del cambio temporal, cultural y lingüístico. Por supuesto, sugiero que no puede ser así. Ni el lenguaje descriptivo ni el lenguaje teorético de una ciencia natural proporciona la base sólida que requeriría esa trascendencia. (T. Kuhn 1990, p. 308).

Los supuestos defendidos por Kuhn lo hacen coincidir con las perspectivas hermenéuticas de la ciencia, las cuales lo estiman como un importante precedente y padrino intelectual. ${ }^{8}$ Sin embargo, Kuhn fue inconsistente con respecto a la relación entre hermenéutica y ciencias naturales. Después de defender el hecho de que las ciencias naturales tienen un fundamento hermenéutico, plantea que las ciencias naturales no son empresas hermenéuticas.

Aunque sostiene que en el contexto de una época determinada, las comunidades científicas asumen un determinado marco conceptual a través de la educación y enmarcado dentro un contexto histórico y cultural, asemejando a

8 Las perspectivas hermenéuticas de la ciencia conciben la ciencia como una actividad interpretativa donde los fenómenos emergen en el contexto de un marco conceptual o una red de significados previos. Las redes de significado emergen de un sistema de prácticas contextualizadas histórica y culturalmente. Estos sistemas de prácticas implican la existencia de un conjunto de habilidades tácitas que posibilitan el aprendizaje y el ejercicio del quehacer científico. Para estas perspectivas cf. P. Heelan 1983; M. Bernstein 1988; R. Rorty 1995; R. Crease (ed.): Hermeneutics and the Natural Sciences 1997. 
las ciencias naturales con las ciencias humanas, Kuhn (2000a) da un giro en la discusión, señalando que a diferencia de lo que ocurre en las ciencias humanas, el marco conceptual proporcionado por la tradición no proporciona en las ciencias naturales una base para la interpretación, sino para la resolución de problemas. Lo que subyace a esta tesis es una vieja concepción de la ciencia que Kuhn había concebido tres décadas antes, en La estructura de las revoluciones científicas.

III. LA NATURALEZA «NO HERMENÉUTICA» DE LA CIENCIA NORMAL

A finales de los años 50s, Kuhn asistió al Center for Advances Studies in the Behavioral Sciences. Su encuentro con los científicos sociales de la institución le permitió observar el marcado desacuerdo existente entre los especialistas de las ciencias sociales sobre cuáles eran sus problemas significativos, los procedimientos y las soluciones para abordar estos problemas. Este desacuerdo motivó la reflexión de Kuhn sobre las razones del consenso en las ciencias naturales. ${ }^{9}$ La respuesta de Kuhn es que las comunidades científicas logran el consenso porque comparten un paradigma. Pero como el término es empleado con tanta ambiguiedad, su solución al problema no es satisfactoria. En uno de los sentidos empleados por Kuhn, el concepto de paradigma remite a una teoría coercitiva que durante un período de tiempo rige la investigación científica. Este período es denominado por Kuhn ciencia normal, la cual define del modo siguiente:

En este ensayo ciencia normal significa la investigación basada firmemente en uno o más logros científicos pasados, logros que una comunidad científica particular reconoce durante algún tiempo como el fundamento de su práctica ulterior. (T. Kuhn 1970, p. 10).

Kuhn entiende la ciencia normal como un proceso de resolución de rompecabezas (puzzle solving). Este concepto es una metáfora fuerte que remite a:

1. Una situación en la que existe una solución prevista a un problema específico que constituye un desafío a la capacidad de los agentes epistémicos.

2. La presencia de un conocimiento tácito en el proceso de resolución de problemas.

3. La carencia de utilidad.

4. Un conjunto de principios no cuestionados que guían la resolución del rompecabezas.

5. La inexistencia de originalidad.

9 Debe recordarse que Kuhn provenía del área de la física. 
El primer aspecto alude al hecho de que, desde la perspectiva de Kuhn, la investigación científica normal es un proceso semejante al juego de resolución de un rompecabezas, donde la solución es una posibilidad lógica y real que sólo la falta de destreza de los individuos impide alcanzar:

Una de las razones por las que la ciencia normal parece progresar tan rápidamente es porque sus practicantes se concentran en problemas que sólo su propia falta de ingenio les impediría resolver. (T. Kuhn 1970, p. 37).

Este proceso carece de un interés directamente práctico, pues constituye más un desafío intelectual que un imperativo social. En uno de sus planteamientos más controversiales Kuhn (1970) sostiene que la ciencia progresa gracias al hecho de que las comunidades científicas son colectividades aisladas que no tienen el imperativo externo a ellas mismas para resolver problemas que tengan una utilidad práctica inmediata. ${ }^{10}$

En otra de las analogías con el juego de rompecabezas, las prácticas científicas son fundamentalmente acríticas. Del mismo modo en que los jugadores no cuestionan nunca los principios que rigen el rompecabezas, los practicantes de las especialidades científicas tampoco cuestionan los fundamentos de la tradición de investigación científica normal que rige sus prácticas de solución de problemas. Si lo hicieran, entonces se asemejarían a los seguidores de escuelas filosóficas y humanísticas donde el permanente cuestionamiento fuerza a una reinvención del estilo, la doctrina o la tradición. ${ }^{11}$

Por el contrario, Kuhn considera que es un requisito imprescindible para el desarrollo y el éxito de la ciencia la ausencia de este tipo de criticidad característica de las humanidades. Gracias a la misma los investigadores pueden concentrarse en el problema proporcionado por su tradición de investigación y hacerla avanzar en el proceso de su resolución.

Kuhn (1970) entiende los procesos críticos o reflexivos como momentos que emergen de las situaciones de crisis. En este sentido, las comunidades científicas de Kuhn intentan resolver sus problemas partiendo de modelos de resolución de problemas no cuestionados. Sólo cuando surgen situaciones anómalas, los modelos de resolución de problemas y las tradiciones en las que se inscriben estos modelos estimulan al cuestionamiento. En la tradición hermenéutica, para autores como Heidegger (1974) y Bollnow (2001) el estado reflexivo surge de una ruptura en la relación práctica con el mundo.

10 Para la crítica a la postura de Kuhn sobre las comunidades científicas como comunidades aisladas, cf. S. Fuller 2000, 2004.

11 Es famoso el debate que generó la crítica a la postura kuhniana sobre la acriticidad de la ciencia, liderada entre otros, por Karl Popper. Sobre el debate cf. I. Lakatos y A. Musgrave (eds.) 1975. 
Por consiguiente, en la perspectiva de Kuhn, el ejercicio cotidiano de la práctica científica no está dirigido a la innovación:

Tal vez el rasgo más sorprendente de los problemas de la investigación normal con el que nos hemos topado hasta ahora sea en cuán escasa medida éstos pretenden producir novedades importantes, sean conceptuales o fenoménicas. En ocasiones, como en la medición de una longitud de onda, se conoce todo por adelantado excepto el detalle más esotérico del resultado, y el margen típico de lo que se espera es sólo ligeramente más amplio [...] la variación de los resultados previstos y por tanto asimilables resulta siempre pequeña comparada con la variación concebible en la imaginación. Además, el proyecto cuyo resultado no cae en este estrecho margen, normalmente no es más que una falla de investigación que refleja no cómo es la naturaleza sino cómo es el científico. (T. Kuhn 1970, p. 35).

En ninguna de las empresas de resolución de problemas características de la ciencia normal ${ }^{12}$ ésta implica el cuestionamiento de los supuestos de la tradición a partir de la cual se definen los hechos relevantes y se establecen los modelos de resolución de problemas. Desde la perspectiva de Kuhn (1970) el éxito de la ciencia normal depende precisamente de la ausencia de la criticidad característica de actividades como la filosofía.

Para Kuhn (2000a), el mecanismo del aprendizaje científico contribuye a esta situación. Este proceso es uno de los signos fundamentales de por qué las ciencias naturales no son empresas hermenéuticas. Debe notarse que para él la noción de hermenéutica remite a interpretación, pero lo más importante, a innovación.

De acuerdo con su tesis del aprendizaje conceptual, Kuhn (1970) entiende el mecanismo mediante el cual se recibe el entrenamiento científico como un proceso de exposición a modelos de resolución de problemas expuestos en libros de textos y bajo la guía de un profesorado. Lo destacable de este proceso además del hecho de que implica la existencia de un conocimiento tácito o inarticulable en un sistema de reglas, es la relación mediatizada que existe entre el estudiantado y la tradición de su especialidad. Contrariamente a lo acontecido en la filosofía, donde el estudiantado requiere del acceso directo a las fuentes originales o los clásicos, el estudiantado de ciencias naturales no requiere leer directamente a los clásicos de su disciplina. Así, mientras es impensable que un estudiante de filosofía o literatura pueda ser un profesional competente sin la lectura directa de Platón o Shakespeare respectivamente, un estudiante de

12 Kuhn señala que la investigación científica normal se sintetiza en tres núcleos básicos de resolución de problemas: La determinación de los hechos relevantes o significativos, la adecuación de los hechos con la teoría predominante y el perfeccionamiento en la articulación de la teoría dominante. Cf. T. Kuhn 1970, pp. 25-28. 
ciencias puede llegar a ser un practicante eficaz de su especialidad sin la necesidad de leer a Galileo, Newton o Darwin.

El proceso de lectura directa de los clásicos fomenta la crítica de fundamentos. Por su parte, el proceso de aprendizaje basado en libros de texto cierra el acceso a las fuentes que originan las tradiciones de investigación impidiendo la lectura crítica de las mismas. Con ello, el mecanismo de enseñanza fomenta la preservación de la tradición antes que la constante reinterpretación característica de los estudios humanísticos.

Por tanto, para Kuhn, el signo distintivo de las comunidades científicas es el ejercicio de las prácticas dirigidas a la autopreservación de la tradición de investigación científica normal. Es este ejercicio de la profesión el que impide, según su perspectiva, la innovación y con ello, que las ciencias naturales sean empresas hermenéuticas. Si las prácticas no estuvieran dirigidas permanentemente a la perpetuación de los fundamentos de la disciplina, entonces la investigación científica estaría caracterizada por un constante enfrentamiento entre escuelas. Se asemejaría al constante proceso de reinvención disciplinar que Kuhn atribuye a la filosofía.

En este contexto, desde la perspectiva de Kuhn, para las ciencias naturales la preservación de los fundamentos de la tradición científica normal es un valor más estimable que la innovación. Contrariamente al filósofo o al artista, valorados en función de su capacidad para crear un estilo, un enfoque o un movimiento intelectual, el científico kuhniano es respetado por su capacidad para resolver los problemas planteados por una tradición que en líneas generales es incuestionada.

Mientras considera a las ciencias humanas como empresas innovadoras, Kuhn entiende que las ciencias naturales no están dirigidas a la innovación. Sólo durante las revoluciones científicas la innovación ocupa un lugar estelar como móvil del progreso cognoscitivo, cuando la tradición de investigación científica normal no puede sobrevivir a la crisis de sus fundamentos, cediendo su lugar a una nueva tradición de investigación. ${ }^{13}$

Por aferrarse a este modelo, Kuhn sostiene la naturaleza no innovadora de las ciencias de la naturaleza frente al carácter innovador de las ciencias humanas, defendiendo que a diferencia de éstas, las prácticas de las ciencias naturales no generan un producto hermenéutico:

Si uno adopta el punto der vista que he estado describiendo respecto a las ciencias naturales, resulta llamativo que lo que hacen principalmente sus profesionales,

13 Debe recordarse que Kuhn (1977b) había señalado que existe una tensión existente entre el pensamiento convergente y el pensamiento divergente de la ciencia. La fase de la ciencia normal, como período no dirigido a la innovación, es la base sobre la cual es posible la ruptura y la innovación que caracteriza la fase de la ciencia post-normal. 
dado un paradigma o base hermenéutica, normalmente no es hermenéutico. (T. Kuhn 2000a, pp. 221-222).

Sin el modelo de ciencia normal-ciencia revolucionaria, Kuhn no se hubiera visto obligado a sostener la contraposición entre preservación e innovación, planteando el desarrollo de las ciencias naturales a partir de una fase de dogmatismo, acriticidad y carencia de innovación opuesta al carácter crítico e innovador de las ciencias humanas.

Esta idea se refuerza tomando en cuenta que, en sus conclusiones, Kuhn se pregunta si la demarcación entre ciencias naturales y ciencias humanas es el producto de un desarrollo histórico o una demarcación basada en la naturaleza de las mismas. Kuhn mismo reconoce su indecisión con respecto a la respuesta, pero afirma que no existe ningún fundamento para sostener que las ciencias humanas no puedan encontrar un paradigma para sustentar la investigación científica normal.

Pero si esto es así, el problema de la demarcación entre ciencias naturales y humanas es un producto histórico basado en la posibilidad o no de la existencia de la ciencia normal. Con lo que se confirma que el apego de Kuhn a esta noción es lo que le lleva a diferir de Taylor, más que en la existencia de la demarcación entre ciencias naturales y ciencias humanas, en el modo de donde debe trazarse la misma.

\section{LAS TENSIONES DE Thomas KuHN}

El problema de la existencia de un período denominado ciencia normal, tan importante para el problema del debate kuhniano con Taylor, se obscurece por la existencia de una tensión existente en la obra de Kuhn: Se trata del contraste entre una concepción de la ciencia como sistema de conocimientos y una concepción de la ciencia como sistema de prácticas.

Kuhn (2000b) consideró su obra como una ruptura con la imagen de la ciencia como un sistema estático de conocimientos y la incorporación de una perspectiva de la ciencia como sistema de prácticas. Esta ruptura significa comprender la ciencia como una serie de actos o acciones (procesos de medición y observación, manipulaciones experimentales, etc.) en que los conceptos, teorías y leyes se aprenden y adquieren sentido, en vez de comprenderla como un sistema estático de creencias del cual se derivan una serie de aplicaciones.

Significa también abandonar el supuesto de que el funcionamiento de la ciencia depende de modo fundamental de la existencia de un sistema algorítmico de reglas y asumir que dicho funcionamiento implica el desarrollo de una serie de destrezas, habilidades o conocimiento tácito, como son los procesos de aprendizaje a partir de modelos de resolución de problemas, o las destrezas para configurar los datos en un laboratorio. 
Aunque Kuhn consideró su obra como una ruptura con la perspectiva de la ciencia como un sistema de conocimientos, realmente, mantuvo una constante tensión entre su propia perspectiva de la ciencia como un sistema de prácticas y la concepción empirista de la ciencia como un sistema de conocimientos. Empleo el término de tensión para referirme a la situación de un sistema conceptual conformado por perspectivas opuestas que generan ambigüedades e inconsistencias en los términos básicos y en el proyecto explicativo en su conjunto.

En La estructura de las revoluciones científicas, Kuhn incorpora una filosofía de las prácticas científicas sin abandonar una concepción de la ciencia como sistema de conocimientos. La tensión generada por esta situación se expresa en un proyecto donde existe una minimización del papel que deben jugar los compromisos teóricos de las comunidades científicas durante el proceso de aprendizaje conceptual que contrasta con la sobreestimación de la función de esos compromisos teóricos como fundamento explicativo de la percepción y de las revoluciones científicas. ${ }^{14}$

$\mathrm{Al}$ incorporar su noción de paradigma como ejemplar, introduce un aspecto innovador en el modo de concebir el desarrollo de la ciencia. El ejemplar es un modelo de resolución de problemas que no se aprende mediante un sistema de reglas. No forma parte de un conocimiento explícito, más bien constituye lo que Polanyi (1964) denomina 'conocimiento tácito', un conjunto de habilidades adquiridas en un proceso de acciones para resolver situaciones problemáticas.

El mecanismo opera como un proceso de reforzamiento y debilitamiento de la conducta. A partir de las soluciones concretas a problemas específicos en el marco de una determinada especialidad, el estudiantado aborda las nuevas situaciones problemáticas relacionándolas con la situación que le ha servido de modelo. Aquellas relaciones de semejanza eficaces para la resolución de los nuevos problemas se refuerzan con respecto a aquellas que se muestran ineficaces.

De acuerdo con este modelo, los conceptos y teorías se derivan de las prácticas, porque se aprenden a partir de ellas.

Kuhn también empleó el término paradigma en el sentido de matriz disciplinar. Con éste término, Kuhn alude a una cosmovisión que contiene los valores o criterios cognitivos para la elección de teorías, las creencias en modelos, las generalizaciones simbólicas y los modelos de resolución de problemas compartidos por los practicantes de una determinada especialidad (ejemplares).

El hecho de colocar los ejemplares dentro de la matriz, indica la preocupación de Kuhn por mostrar este conjunto de compromisos como un todo en

14 Aunque Bird (2000) habla de una tensión entre la sobrestimación de la teoría y la minimización de la misma, no percibe la misma como el producto de la tensión existente en las dos concepciones filosóficas señaladas en este artículo. 
el que interactúan los distintos componentes. De hecho, al definir la matriz disciplinar, Kuhn señala que sus componentes conforman una totalidad y que funcionan unitariamente. No obstante, como expresión de la tensión entre sus dos concepciones filosóficas, Kuhn no es consecuente con este planteamiento, porque en su proyecto explicativo hay una clara desvinculación de estos componentes, priorizando la función que desempeñan los ejemplares para el proceso de percepción y del aprendizaje conceptual.

Dependiendo de cómo se utilice el término paradigma, este implicará consecuencias importantes para explicar los problemas que Kuhn intentó solucionar en La estructura, como la cuestión del consenso. Si se recurre al término de paradigma en su acepción de matriz disciplinar, entonces las comunidades científicas logran el acuerdo en base al hecho de compartir una cosmovisión del mundo. En este sentido, las comunidades científicas no se distinguen de otras comunidades como las de los filósofos, los teólogos, o los integrantes de un determinado movimiento estético.

Pero si el término se emplea en el sentido de ejemplar, entonces la causa del consenso de las comunidades científicas no viene dado por el hecho de compartir cosmovisiones o sistema de creencias. Por el contrario, el consenso viene dado por compartir un quehacer. Esto daría la razón a Doppelt (1978) y a Rouse (2003), para quien las comunidades científicas de Kuhn no comparten una ortodoxia, sino problemas y técnicas, en síntesis, un sistema de prácticas.

Debido a las ambigüedades de Kuhn, la tesis de Rouse no es fácil de confirmar. Kuhn parece decantarse por las teorías como determinantes en el consenso de las comunidades científicas cuando en el segundo capítulo de $L a$ estructura, se refiere a la situación de desacuerdo característico del período de la óptica pre-newtoniana.

No obstante, en la postdata a La estructura de las revoluciones científicas, Kuhn establece cómo los ejemplares producen un consenso disciplinar independientemente del disenso teórico.

El problema del consenso es básico para posibilitar la ciencia normal. Desde una perspectiva de la ciencia como sistema estático de conocimientos, la ciencia normal requeriría de la existencia de un cuerpo teórico que proporcione el consenso y fundamente la investigación. Kuhn (1970) habla de la existencia de compromisos teóricos que se constituyen en paradigmas guían de modo coercitivo la investigación científica normal.

Si en la imagen de la ciencia como sistema estático de conocimientos las prácticas se derivan del conjunto de las teorías predominantes, y los conceptos se aprenden como parte de una fase previa al proceso de las aplicaciones, en la filosofía de las prácticas científicas de Kuhn, el proceso es inverso, las teorías se aprenden en el contexto de un quehacer y los conceptos adquieren sus sig- 
nificados dentro del marco del proceso de las observaciones y actividades de las comunidades científicas.

Sin embargo, la idea de que las teorías dependan de los procesos situacionales inherentes a las prácticas científicas es inconsistente con la tesis kuhniana de la percepción y del cambio conceptual. En una filosofía de las prácticas científicas, la percepción no se ve como un proceso determinado por las teorías, la percepción es más bien un fenómeno más de las prácticas científicas donde los instrumentos y los sistemas tecnológicos integran a los marcos conceptuales. De hecho, una filosofía de las prácticas científicas puede articularse prescindiendo de una noción fuerte de teoría como parte del proceso observacional. Un ejemplo de ello es la perspectiva de Ian Hacking (1996).

Al hacer de las teorías determinantes de la percepción, Kuhn genera paradojas para su proyecto explicativo. Por una parte, la percepción forma parte de un proceso de aprendizaje de relaciones de semejanza y disimilitud que no requiere del aprendizaje de un sistema de reglas ni de conocimientos explícitos como son las teorías. Por otra parte, la percepción de los científicos depende de las teorías de tal modo que si éstas se modifican, transforman el objeto mismo de percepción.

Al mismo tiempo, la tensión existente entre su filosofía de las prácticas científicas y su concepción de la ciencia como sistema estático de conocimientos, genera también una ambigüedad importante para la tesis kuhniana del cambio conceptual. Compromisario de una filosofía de las prácticas científicas es lógico que Kuhn conciba las revoluciones científicas como transformaciones en los ejemplares de una determinada tradición de investigación. Pero cuando leemos el capítulo X de La estructura, sale a relucir que Kuhn entiende las revoluciones científicas básicamente como cambios de cosmovisión (paradigmas matrices) o en términos de cambio teórico. Kuhn entiende que tras una revolución científica, los libros de texto deberán ser reescritos para hacerlos acorde con la nueva tradición de ciencia normal. Es en estos libros de texto donde se exponen los ejemplares que, a juicio de Kuhn (1977), modelan las mentes de los futuros científicos generando sus predisposiciones mentales.

Pero, si los libros de texto son reescritos a partir de las transformaciones conceptuales, entonces los modelos de resolución de problemas dependen fundamentalmente de las teorías y cosmovisiones, con lo que el concepto básico llamado a fundamentar la filosofía de las prácticas científicas de Kuhn depende de las nociones clásicas de una perspectiva de la ciencia como sistema estático de conocimientos.

En conclusión, el proyecto explicativo de Kuhn se ve notablemente afectado por las tensiones constitutivas de su obra, lo que explica tanto las ambigüedades conceptuales de su teoría de la ciencia, así como la diversidad de interpreta- 
ciones contradictorias que generó para su debate posterior con Taylor y para su legado.

\section{A MODO DE CONCLUSIÓN}

Luego de confrontar a Taylor por su asunción de la dicotomía entre ciencias naturales y ciencias humanas, Kuhn mostró ambigüedades respecto a la existencia de esta demarcación. Después de considerar que las ciencias naturales tienen una base hermenéutica, consideró que no son por sí mismas empresas hermenéuticas. Esta paradoja se explica por los compromisos de Kuhn con su proyecto explicativo planteado casi tres décadas antes en La estructura de las revoluciones científicas.

Estos compromisos están vinculados con la existencia de una tensión entre una concepción de la ciencia como sistema de conocimientos y una imagen de la ciencia como sistema de prácticas. Esta tensión obscurece muchos de los problemas planteados por Kuhn, como el de la naturaleza del consenso, los paradigmas y con ello, la naturaleza misma de la ciencia normal con sus respectivas consecuencias para la concepción kuhniana de la misma para su debate con Taylor.

Insatisfecho con los fundamentos de la demarcación entre ciencias naturales y ciencias humanas, Kuhn no propone una alternativa coherente. Si bien parece considerar que las ciencias naturales tienen suficientes semejanzas con las ciencias humanas para ser consideradas actividades hermenéuticas, concluye retomando la distinción sobre la base de la asunción de su viejo modelo dicotómico entre ciencia normal y ciencia revolucionaria que replantea la demarcación de Taylor sobre nuevas bases. Al hacerlo, Kuhn vuelve a acercarse a la tradición del empirismo lógico con la que usualmente se le ha confrontado, aceptando que la demarcación entre ciencias naturales y ciencias humanas debe verse más como un producto histórico que como un resultado de la diferencia en la naturaleza misma de las ciencias. Como consecuencia, Kuhn está más cerca de la tesis empirista de la unidad del saber, que de la tradición hermenéutica que ha intentado mostrar una diferencia en la naturaleza entre ciencias naturales y humanas, o entre los más modernos enfoques hermenéuticos que han asumido relaciones de semejanza entre ambas sobre la base de la existencia de una dimensión hermenéutica entre todas las ciencias.

De este modo, el legado de Kuhn a cinco décadas de la publicación de $L a$ estructura de las revoluciones científicas no deja de ser paradójico. Considerado como un padrino intelectual de los recientes programas hermenéuticos, Kuhn asumió tesis que le acerca y le distancia de estos programas. Su asunción de que las ciencias naturales abordan significados y que están determinadas histórica y culturalmente, así como la asunción de la ciencia basada en un sistema 
de prácticas que implican una dimensión de conocimiento tácito, le acercan notablemente a los enfoques hermenéuticos.

No obstante, su concepción de la ciencia regida por un período coercitivo llamado ciencia normal, la idea de que las ciencias naturales se basan más en la preservación de la tradición que en la innovación y su tesis sobre la demarcación entre ciencias naturales y ciencias humanas fundamentada en la noción de ciencia normal, lo colocan en las antípodas de estos enfoques.

\section{REFERENCIAS BIBLIOGRÁFICAS}

BERSTEIN, M. 1998: Beyond Objetivism and Relativism: Science, Hermeneutics and Praxis. Philadelphia, Pennsylvania: University of Pennsylvania Press.

BIRD, A. 2000: Thomas Kuhn. Princeton, N.A.: Princeton University Press.

BOLLNOW, O. 2001: Introducción a la Filosofía del Conocimiento: La comprensión previa y la experiencia de lo nuevo. Buenos Aires: Amorrortu Editores.

CREASE, R. (ed.) 1997: Hermeneutics and the Natural Sciences. Dordrecht: Kluwer Academic Publishers.

FULLER, S. 2000: Thomas Kuhn: A Philosophical History for our Time. Chicago, Illinois: The University of Chicago Press.

FULLER, S. 2004: Kuhn vs. Popper: The Struggle for the Soul of Science. New York: Columbia University Press.

GADAMER, H. 1997: Verdad y método (2 vols.) Salamanca: Ed. Sígueme.

GATTEI, S. 2008: Thomas Kuhn's «linguistic turn» and the Legacy of Logical Empirism: Incommensurability, Rationality and the Search for Truth, Aldershot, Hampshire: Ashgate Publishing Limited.

HACKING, I. 1996: Representar e intervenir. México, D.F: Ed. Paidós.

HANSON, N. 1977: Patrones de Descubrimiento. Montesinos. Madrid: Alianza Editorial.

HEIDEGGER, M. 1974: El Ser y el Tiempo. México, D.F: FCE.

HEELAN,P. 1983: «Natural Science as a Hermeneutic of Instrumentattion», Philosophy of Science, 50, pp. 181-204.

HOYNINGUEN HUENE, P. 1998: Reconstructing Scientific Revolutions: Thomas S. Kuhn's Philosophy of Science. Chicago, Illinois: The University of Chicago Press.

LAKATOS, I. y MUSGRAVE, A. (eds.) 1970: Criticism and the growth of knowledge. Cambridge: Cambridge University Press.

KUHN, T. 1970: The structure of scientific revolutions. Chicago, Illinois: The University of Chicago Press.

KUHN, T. 1977a: Objectivity, value judgment, and theory choice. En T. Kuhn (Ed.), The essential tension: Selected studies in scientific tradition and change. Chicago, Illinois: The University of Chicago Press, pp. 320-339.

KUHN, T. 1977b: «The essential tension: Tradition and innovation in scientific research», en T. Kuhn, The essential tension: Selected studies in scientific tradition and 
change. Chicago, Illinois: The University of Chicago Press, pp. 225-239.

KUHN, T. 1990: «Dubbing and redubbing: The vulnerability of rigid designation», en C.W. Savage (ed.) Scientific theories. Minneapolis: University of Minnesota Press.

KUHN T. 2000a: «The natural and the human sciences», en J. Conant y J. Haugeland (eds.) The road since structure. Chicago, Illinois: Chicago University Press, pp. 216-223.

KUHN, T. 2000c: The problem with the historical philosophy of science. En J. Conant y J. Haugeland (eds.), The road since structure. Chicago, Illinois: The University of Chicago Press, pp. 105-120.

OLIVE, L. y PEREZ RANSANZ, A.R. (eds.) 1998: Filosofía de la Ciencia: Teoría y Observación. México, D.F: FCE

OLIVE, L 1998.: «Constructivismo, Relativismo y Pluralismo en la Filosofía y Sociología de la Ciencia», en: C. Solís Santos (eds.): Alta tensión: Historia, filosofía y sociología de la Ciencia: Ensayos en Memoria de Thomas Kuhn: Barcelona, Ed. Paidós, pp.195-211.

PEREZ RANSANZ, A.R. y ÁLVAREZ, J.F. 2004: «De Kant a Kuhn, acotando por Putnam», Endoxa, Series Filosóficas, 18, pp. 495-517.

POLANYI, M. 1964: Personal Knowledge: Towards a post-critical Philosophy. New York, N.Y: Harper and Row.

RORTY, R. 1995: La Filosofía y el Espejo de la Naturaleza. Madrid: Cátedra.

SANKEY, H. 1993: «Kuhn's Changing Concept of Incommensurability», British Journal for the Philosophy of Science, 44 (4), pp. 775-791.

TAYLOR, Ch. 1985: «Interpretation and the Sciences of Man», en Philosophy and the Human Sciences. Cambridge: Cambridge University Press, pp. 15-57.

Leonardo Díaz es Profesor Adjunto del departamento de Filosofía de la Universidad Autónoma de Santo Domingo (UASD) y miembro de Número de la Unidad de Epistemología de la Academia de Ciencias de la República Dominicana.

Líneas de investigación:

Estudios CTS, Epistemología (Thomas Kuhn, Karl Popper), sociedad de la información y del conocimiento.

Publicaciones recientes:

(2011) "Las tensiones de La estructura de las revoluciones científicas y el legado de Thomas Kuhn”, Endoxa, vol. 27, pp. 251-270.

(2012) Las tensiones de Thomas Kuhn. Perspectiva crítica para los estudios sociales y culturales de la ciencia (Libro aprobado para su publicación).

Dirección electrónica: rld_do@yahoo.com 